\title{
Diversidad y composición de coleópteros del Parque Nacional Bernardo O’Higgins (región de Magallanes, Chile)
}

\author{
Diversity and composition of beetles of Bernardo O'Higgins National Park \\ (region of Magallanes, Chile)
}

\author{
Christian Muñoz-Escobar ${ }^{\text {a,b }}$, Viviane Jerez ${ }^{\text {b* }}$ \\ a Universidad de Concepción, Programa de Doctorado en Sistemática y Biodiversidad, Concepción, Chile. \\ *Autor de correspondencia: ${ }^{\mathrm{b}}$ Universidad de Concepción, Facultad de Ciencias Naturales y Oceanográficas, \\ Departamento de Zoología, Barrio Universitario s/n, Concepción, Chile, tel.: 56-412204965, vijerez@udec.cl
}

\section{SUMMARY}

Bernardo O'Higgins National Park (BONP) is the largest protected area in Chile and southern hemisphere. With over 3.5 million hectares, it has a complex continental and archipelagic topography with a mosaic of evergreen forests, coastal scrub and peatlands. Given the future of the Chilean environmental legislation, all protected areas will be re-evaluated according to scientific parameters. Therefore, and considering that insects are currently recognized by the Chilean law, we aim at providing information on the spatial distribution of beetles using pitfall traps in 20 sites associated with the park. We evaluate the effect of vegetation and type of environment (insular, continental) on taxonomic composition, richness, abundance and diversity of beetles. A total of 15 families, 33 genera and 38 species were recorded. Many of these represent monotypic groups. The most diverse families were Carabidae, Staphylinidae and Curculionidae. No significant effect of vegetation and type of environment on any community measurement was observed since the vegetation and sectors analyzed are part of a larger biogeographic unit. Additionally, we determined 11 species to the park, which are significantly associated among them. We discuss the importance of this kind of information to make biogeographical approaches.

Key words: Coleoptera, patagonian channels, magellanic paramo, kawésqar, Chile.

\section{RESUMEN}

El Parque Nacional Bernardo O’Higgins (PNBO), corresponde al área silvestre protegida más extensa de Chile y del Hemisferio Sur; con más de 3,5 millones de hectáreas presenta una compleja topografía continental e insular con un mosaico de bosques perennifolios, matorral costero y turberas. Considerando que los insectos son actualmente reconocidos por la legislación chilena, el objetivo fue entregar información de la distribución espacial de coleópteros capturados con trampas de intercepción en 20 sitios asociados al parque y evaluar el efecto que tiene la vegetación y tipo de ambiente (insular, continental) sobre la composición taxonómica, riqueza, abundancia y diversidad de los mismos. Se registró un total de 15 familias, 33 géneros y 38 especies, muchas de las cuales pertenecen a géneros monotípicos. Las familias más diversas fueron Carabidae, Curculionidae y Staphylinidae. No se evidenció efecto significativo del tipo de ambiente ni de la vegetación sobre ningún parámetro comunitario analizado; lo anterior respondería a que las formaciones vegetales y sitios investigados forman parte de una unidad biogeográfica mayor. Por otra parte, se determinó que 11 taxones se encuentran asociados significativamente. Se discute, la importancia de este tipo de información al momento de realizar aproximaciones biogeográficas.

Palabras clave: Coleoptera, canales patagónicos, páramo magallánico, kawésqar, Chile.

\section{INTRODUCCIÓN}

Actualmente, Chile protege alrededor del $19 \%$ de su territorio continental (Jorquera-Jaramillo et al. 2012) y del cual cerca del $84 \%$ se encuentra en las regiones australes del país (Pauchard y Villarroel 2002, Jorquera-Jaramillo et al. 2012), principalmente en áreas insulares de difícil acceso y sin perturbación antrópica. La biota de estos sectores no presenta una gran diversidad biológica como ocurre en bosques tropicales (Jerez y Muñoz-Escobar 2015); no obstante, posee gran diversidad de especies endémicas de ori- gen gondwánico, cuyos patrones actuales de distribución son resultado de eventos glaciales pleistocénicos (Crisci et al. 1991).

Uno de los grupos más característicos de la biota austral corresponde a insectos coleópteros que presentan géneros endémicos como Antarctonomus, Cascellius, Ceroglossus y Creobius (Carabidae) y Aegorhinus, Alastoropolus, Antarctobius, Falklandius y Telurus (Curculionidae) entre otros (Morrone et al. 1994, Roig-Juñent 2000, Jerez y Muñoz-Escobar 2015). Estos taxones han sido fundamentales para la formulación de esquemas biogeográficos que 
definen parte de las provincias biogeográficas del Páramo Magallánico y Bosque Magallánico de la sub-región Subantártica (Morrone 2006). Sin embargo, este tipo de organismos rara vez han sido considerados para caracterizar o evaluar los ambientes naturales de Chile, producto de la escasa o nula información que existe de ellos principalmente al sur del país (Jerez et al. 2015, Jerez y MuñozEscobar 2015).

Estos últimos años, Chile ha experimentado una serie de cambios relacionados a su institucionalidad ambiental. Es así que producto de la promulgación de la Ley 20.417, en el año 2010 se crea el Ministerio del Medio Ambiente (MINSEGPRES 2010), se rediseña la orgánica ambiental chilena y se modifica el artículo 37 de la Ley 19300 (MMA 2011), permitiendo ahora clasificar bajo alguna categoría de conservación a insectos y otros invertebrados (Jerez et al. 2015). Por otra parte, el Proyecto de Ley para la creación del Servicio de Biodiversidad y Áreas Protegidas y el Sistema Nacional de Áreas Protegidas (actualmente en trámite legislativo), contempla la realización y actualización de inventarios de especies (MMA 2015). Esto último, repercutirá en la necesidad de recopilar información sobre la diversidad, distribución geográfica y ecología de los insectos en los diversos ecosistemas del país.

En Chile, los coleópteros están representados por 4.200 especies que corresponden a un $30 \%$ del total de especies de insectos descritas para Chile (Elgueta 2008). Sin embargo, para la región de Magallanes no han sido frecuentes los estudios que analicen la riqueza de especies de coleópteros, junto a aspectos distribucionales y su relación con regiones ecológicas basadas por ejemplo en formaciones vegetacionales. El caso de esta región es anecdótico, ya que a pesar de contar con más de un $50 \%$ de superficie bajo alguna categoría de área protegida (Pauchard y Villarroel 2002), poco se conoce de los patrones de distribución de las especies que allí habitan, producto de la restricción de acceso a redes viales, generando que muchos sectores en Magallanes sigan inexplorados (Jerez y Muñoz-Escobar 2015). Hasta la actualidad la información sobre coleópteros es fragmentaria y dispersa, faltando un análisis de conjunto de la biodiversidad, su distribución y relación con las formaciones vegetacionales presentes en el área. Por otra parte, los registros de presencia de muchas especies, se basan en localidades sin coordenadas geográficas, sesgadas a sectores estepáricos o bien a áreas de interés turístico y de fácil acceso, aledaños al Parque Nacional Torres del Paine.

El Parque Nacional Bernardo O'Higgins (PNBO), es uno de los parques más inhóspitos e inexplorados de Chile; se encuentra localizado entre las regiones de Aysén y Magallanes $\left(47^{\circ} 55^{\prime} \mathrm{S}-51^{\circ} 37^{\prime} \mathrm{S}\right)$ y representa el área silvestre protegida con mayor superficie del Hemisferio Sur, siendo al mismo tiempo el área de conservación ecocultural más importante de Sudamérica (CEQUA 2011). Este parque presenta una vegetación dominada por turberas (Pisano 1977) en condiciones extremas de humedad, vien- to y frío dada la influencia del océano Pacífico (CEQUA 2011). Dado lo anterior, las comunidades bióticas presentes en el parque debiesen ser resultado de procesos abióticos relativamente recientes, con la presencia de especies adaptadas a condiciones de aislamiento. En este sentido, el conocimiento de su biota puede contribuir a la protección de este tipo de ecosistema y por ello los inventarios de especies constituyen un punto de partida para comprender el funcionamiento de los sistemas ecológicos y el posterior desarrollo de políticas de conservación (Jerez y MuñozEscobar 2015, Jerez et al. 2015). Por tanto, es importante recopilar, resumir y asociar información tanto de la composición de especies como su distribución geográfica. Así, se facilita y dirige el desarrollo de planes de manejo en áreas protegidas, al evaluar la relación de especies con un ambiente determinado y definir cuáles son y el grado de asociación que existe entre las especies que modulan la estructuración espacial.

Los coleópteros establecen con la vegetación relaciones ecológicas muy variadas, que van desde el uso del sitio como refugio, búsqueda de presas, herbívora y/o descomposición de materia orgánica (Jerez y Muñoz-Escobar 2015), factores que convierten a estos insectos como buenos bioindicadores de tipos de hábitats o ambientes. Dado lo anterior, se espera encontrar una estructuración espacial en la diversidad y composición taxonómica de coleópteros en función de las formaciones vegetacionales (e.g. bosque, matorral, turberas) y tipo de ambientes (e.g. insular, continental) presentes en el PNBO. Por ello, los objetivos del presente estudio son 1) caracterizar la diversidad y composición taxonómica de coleópteros presentes en el PNBO y compararla en función de las formaciones vegetacionales y tipos de ambiente (insular vs. continental) allí presentes, y 2) determinar qué especies de coleópteros son las que mejor podrían describir un patrón de ordenamiento.

\section{MÉTODOS}

Sitios y método de muestreo. Los muestreos se efectuaron en el Parque Nacional Bernardo O'Higgins (PNBO), en 20 sitios ubicados entre el Glaciar Jorge Montt y Fiordo Encuentros, en relación a las formaciones vegetacionales allí presentes: Bosque Magallánico Perennifolio, Matorral Costero y Complejo de Tundra Magallánica (Pisano 1977) y 33 unidades experimentales: Bosque, $\mathrm{N}=18$; Matorral, $\mathrm{N}=9$; y Turbera, $\mathrm{N}=6$ (figura 1).

Los muestreos se realizaron en noviembre de 2009 y enero de 2010. Los coleópteros se capturaron utilizando trampas de intercepción, las cuales consistieron en recipientes plásticos de 10 centímetros de diámetro y 15 centímetros de alto, enterrados a ras de suelo y rellenados con una mezcla de alcohol y agua $\left(70^{\circ}\right)$ como líquido preservante (Koivula et al. 2003). Este método de muestreo con trampas de intercepción está dirigido principalmente a artrópodos caminadores y se consideró como el más adecuado para este tipo de ambiente, con condiciones extremada- 


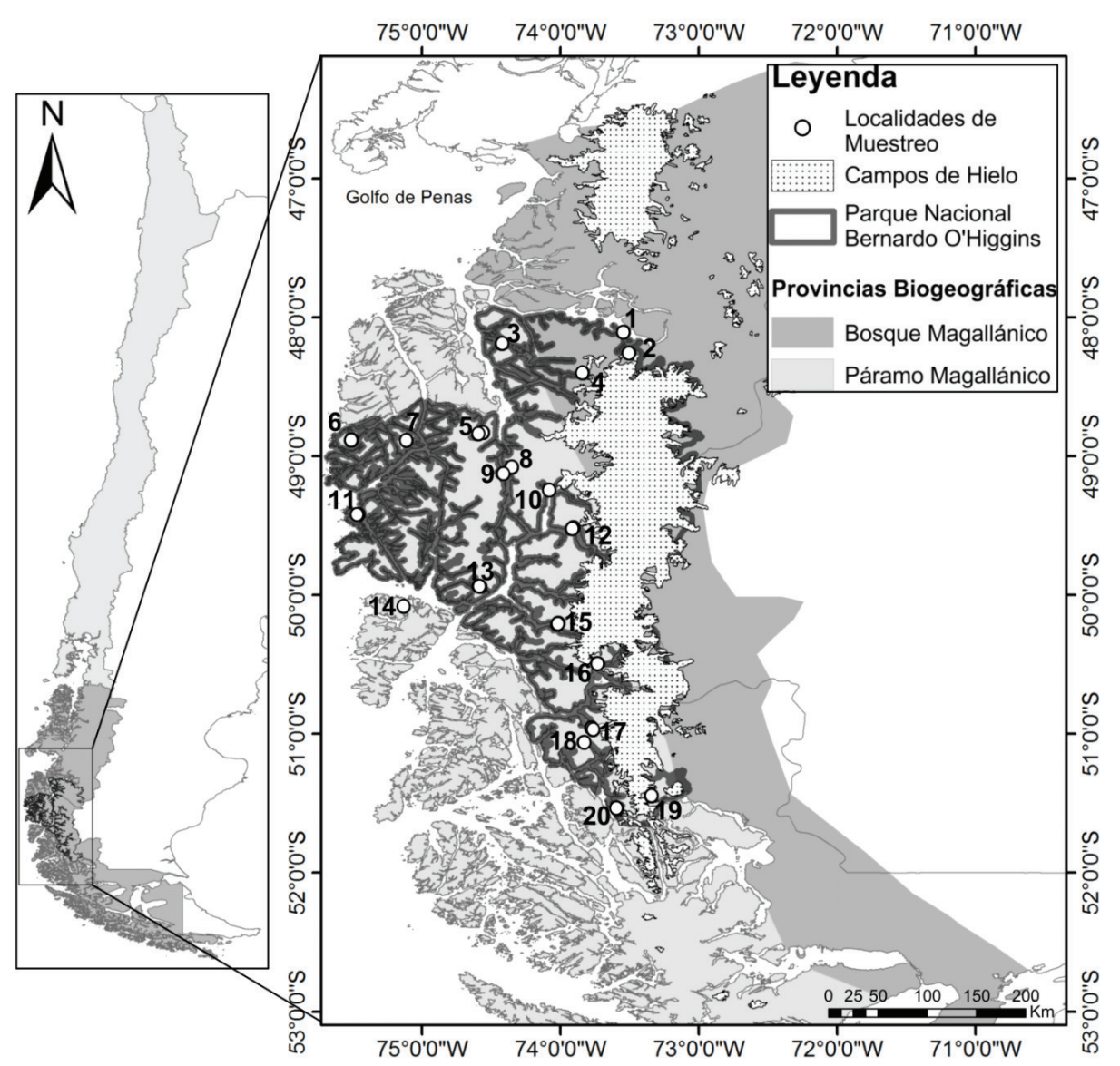

Figura 1. Sitios de Muestreo del PNBO: 1) Isla San Francisco; 2) Glaciar Jorge Montt; 3) Isla Van Der Meulen; 4) Fiordo Ofhidro; 5) Seno Wald; 6) Isla Esmeralda; 7) Isla Orella; 8) Bahía Edimburgo; 9) Puerto Edén; 10) Glaciar Pío XI; 11) Isla Kalau; 12) Fiordo Falcón; 13) Seno White; 14) Isla Madre de Dios; 15) Estero Europa; 16) Estero Asia; 17) Glaciar Amalia; 18) Fiordo Norte; 19) Quebrada Puma; 20) Fiordo Encuentros.

Sampling locations in the BONP: 1) San Francisco Island; 2) Jorge Montt Glacier; 3) Van Der Meulen Island; 4) Ofhidro Fjord; 5) Wald Fjord; 6) Esmeralda Island; 7) Orella Island; 8) Edimburgo Bay; 9) Puerto Eden; 10) Pio XI Glacier; 11) Kalau Island; 12) Falcon Fjord; 13) White Fjord; 14) Madre de Dios Island; 15) Europe Fjord; 16) Asia Fjord; 17) Amalia Glacier; 18) North Fjord; 19) Quebrada Puma; 20) Encuentros Fjord.

mente inhóspitas tanto climáticas (lluvia y vientos permanentes) como logísticas (factibilidad de acceso a sectores insulares, condiciones de navegación).

En cada unidad experimental, se instalaron 30 trampas divididas en grupos de tres, las que fueron separadas unas de otras por $5 \mathrm{~m}$ intra-grupos y $15 \mathrm{~m}$ inter-grupos, lo que constituyó un total de 10 unidades muestrales. Para el total de los muestreos, se instalaron 990 trampas las que por factibilidad logística (i.e. logísticas de terreno y condiciones meteorológicas) operaron por 24 horas aproximadamente. Se eligió trabajar con grupos de trampas, considerando la naturaleza en mosaico de este tipo de ambiente y así aumentar la probabilidad de capturar insectos.

Procesamiento de muestras e identificación de taxones. Una vez en el laboratorio, todo el material capturado fue separado, etiquetado y conservado en frascos con alcohol de $70^{\circ}$ hasta el momento de su recuento y determi- nación taxonómica. Los insectos fueron separados bajo lupa estereoscópica y posteriormente montados en alfileres entomológicos o conservados en frascos con alcohol $70^{\circ}$, etiquetados e ingresados a la colección entomológica del Museo de Zoología de la Universidad de Concepción (UCCC).

Para la clasificación de las categorías taxonómicas de Coleoptera se consultó literatura especializada como Jeannel (1962), Roig-Juñent (2000) y Jiroux (2006), entre otros. La determinación se ratificó además por comparación con material conservado en colecciones de referencia pertenecientes a la Universidad de Concepción.

Análisis comunitarios. Para resumir la complejidad de los ensamblajes de coleópteros, en cada unidad experimental, se calculó la riqueza específica (S) y abundancias $(\mathrm{N})$. Además se estimaron los índices de exponencial de Shannon (exp H') (Jost 2006) e inverso de Gini-Simpson 
$\left(1 / 1-\lambda^{\prime}\right)$ (Jost 2006), los cuales modifican los clásicos índices de entropía de Shannon (Shannon 1948) y Simpson (Simpson 1949) respectivamente, transformándolos en números efectivos de especies, equivalente a una medida de verdadera diversidad (Jost 2006). Por cada parámetro comunitario, se construyó una matriz de distancia Euclidiana entre pares de observaciones, con el objeto de evaluar diferencias entre los tipos de ambiente (insular y continental) y formaciones vegetacionales (bosque, matorral, turbera), utilizando análisis de varianza basada en permutaciones (PERMANOVA). Este procedimiento es equivalente a un análisis de varianza estándar, pero la flexibilidad y robustez de una aproximación basada en permutaciones utilizando el algoritmo de Montecarlo (MC), aminora la necesidad que las variables cumplan con los supuestos de normalidad (Anderson et al. 2008).

Para evaluar la estructuración en la composición de coleópteros entre los tipos de ambientes y formaciones vegetacionales, se realizó un PERMANOVA. Dada la dominancia de dos a tres especies a lo largo de todo el ensamblaje, las abundancias de las especies fueron transformadas a raíz cuarta y así otorgar poder de explicación a las especies raras (Clarke y Gorley 2006). Para representar y contrastar gráficamente el patrón distribucional de coleópteros, se realizó un escalamiento multidimensional no paramétrico (nMDS) sobre la matriz de similitud de BrayCurtis (Clarke et al. 2006) y Sørensen (Sørensen 1948), basadas en las abundancias y presencia/ausencia de las especies respectivamente. Posteriormente, se definió un subconjunto de especies que mejor describen la coleopterofauna registrada en el PNBO. Para ello se aplicó el algoritmo paso a paso BVSTEP (Clarke y Warwick 1998), el cual mide la correlación ( $\rho$ ) a través del coeficiente de Spearman, entre la matriz general y un subconjunto de especies seleccionado, hasta alcanzar al menos un valor de $\rho$ superior a 0,95 .

Por otro lado, se evaluó la asociación entre las especies de coleópteros registradas en el PNBO. Para ello, se utilizó el índice de asociación de Whitaker (Whittaker 1952) para efectuar un análisis inverso modo- $\mathrm{R}$ entre las variables (especies) de los diversos sitios de muestreo. Primero se realizó un análisis de perfil de similitud (SIMPROF) tipo 2 (Somerfield y Clarke 2013), el cual utilizando permutaciones evaluó de manera global si existe asociación de especies en la estructura multivariada del ensamblaje en comparación a lo esperado por azar. Posteriormente, con el objeto de determinar que especies forman "grupos coherentes" o asociados significativamente $(P>0,05)$, se realizó un SIMPROF tipo 3 (Somerfield y Clarke 2013) considerando aquellas especies del subconjunto de especies que explican el patrón de ordenamiento global (estimados con BVSTEP). Todos los análisis tanto en modo$\mathrm{Q}$ (entre muestras) como en modo-R (entre especies), se realizaron con los programas PRIMER-E v6.1.12 (Clarke y Gorley 2006) y PERMANOVA+ v1.0.2 (Anderson et al. 2008).

\section{RESULTADOS}

Diversidad y composición taxonómica. Para el PNBO se capturaron 347 coleópteros, clasificados en 15 familias, 33 géneros y 38 especies (cuadro 1). Las familias con mayor número de especies fueron Carabidae $(\mathrm{n}=9)$, Curculionidae $(\mathrm{n}=8)$ y Staphylinidae $(\mathrm{n}=7)$, mientras que las familias más abundantes fueron Carabidae $(\mathrm{n}=237)$, Cryptophagidae $(\mathrm{n}=24)$ y Curculionidae $(\mathrm{n}=20)$. Las especies más abundantes fueron los carábidos Ceroglossus suturalis (Fabricius, 1775), $(\mathrm{n}=159)$ y Cascellius septentrionalis Roig-Juñent, 1995, $(\mathrm{n}=46)$. Sólo cinco especies están presentes en las tres formaciones vegetacionales: C. septentrionalis y C. suturalis (Carabidae), Nothofaginoides andinus Kuschel, 1952 (Curculionidae), Nemadiopsis sp. (Leiodidae) y Cyphon sp. (Scirtidae) (cuadro 2). Al analizar los distintos parámetros comunitarios, no se observó un efecto significativo del tipo de ambiente y tipo de formación vegetacional (cuadro 3). En promedio, por sitio de muestreo se obtuvo una riqueza específica de 3,78 $\pm 0,39$ y una abundancia de $10,84 \pm 1,6$; mientras que los valores promedio de diversidad del exponencial de Shannon fue de 3,04 \pm 0,32 e inverso de Gini-Simpson fue de $2,70 \pm 0,29$ (figura 2).

Similitud biótica entre sitios de muestreo. Al evaluar la estructuración de coleópteros a lo largo del PNBO, no se observaron diferencias considerando el tipo de ambiente y formación vegetacional (cuadro 3). A través de BVSTEP, se determinó que 11 especies representan el patrón global del ensamblaje de coleópteros presentes en el PNBO $(\mathrm{P}=0,95$; $P<0,001)$; estas fueron los Carabidae: C. suturalis, C. septentrionalis, Creobius eydouxii (Guérin-Ménéville, 1838) y Nothocascellius hyadesii (Fairmaire, 1885), Cryptophagidae: Chiliotus sp., Curculionidae: Nothofaginoides andinus, Hydrophilidae: Cercyon quisquilius (Linnaeus, 1761), Leiodidae: Nemadiopsis sp., Melandryidae: Orchesia sp., Scirtidae: Cyphon sp. y Staphylinidae: Cheilocolpus magallanicus Sáiz, 1970. La mayoría de estas especies son más abundantes en sectores de la Isla Wellington $\left(49^{\circ}-50^{\circ} \mathrm{S}\right)$ y áreas continentales subyacentes (figura 3).

Por otro lado, al analizar las correlaciones interespecíficas a través del test SIMPROF tipo 2, se determinó una asociación significativa entre especies $(\pi=0,021 ; P$ $<0,001)$. De esta forma, al considerar el subconjunto de especies "más importante" obtenidos con BVSTEP, se obtuvo según la prueba SIMPROF tipo 3 que todas estas covariaron coherentemente a lo largo y ancho del PNBO formando una sola asociación genuina (figura 4).

\section{DISCUSIÓN}

Los resultados del presente estudio indican una baja riqueza específica en comparación a lo descrito para el territorio entre $48^{\circ}$ y $51^{\circ}$ lat $\mathrm{S}$ (Jerez y Muñoz-Escobar 2015). Sin embargo, como característica de los coleópte- 


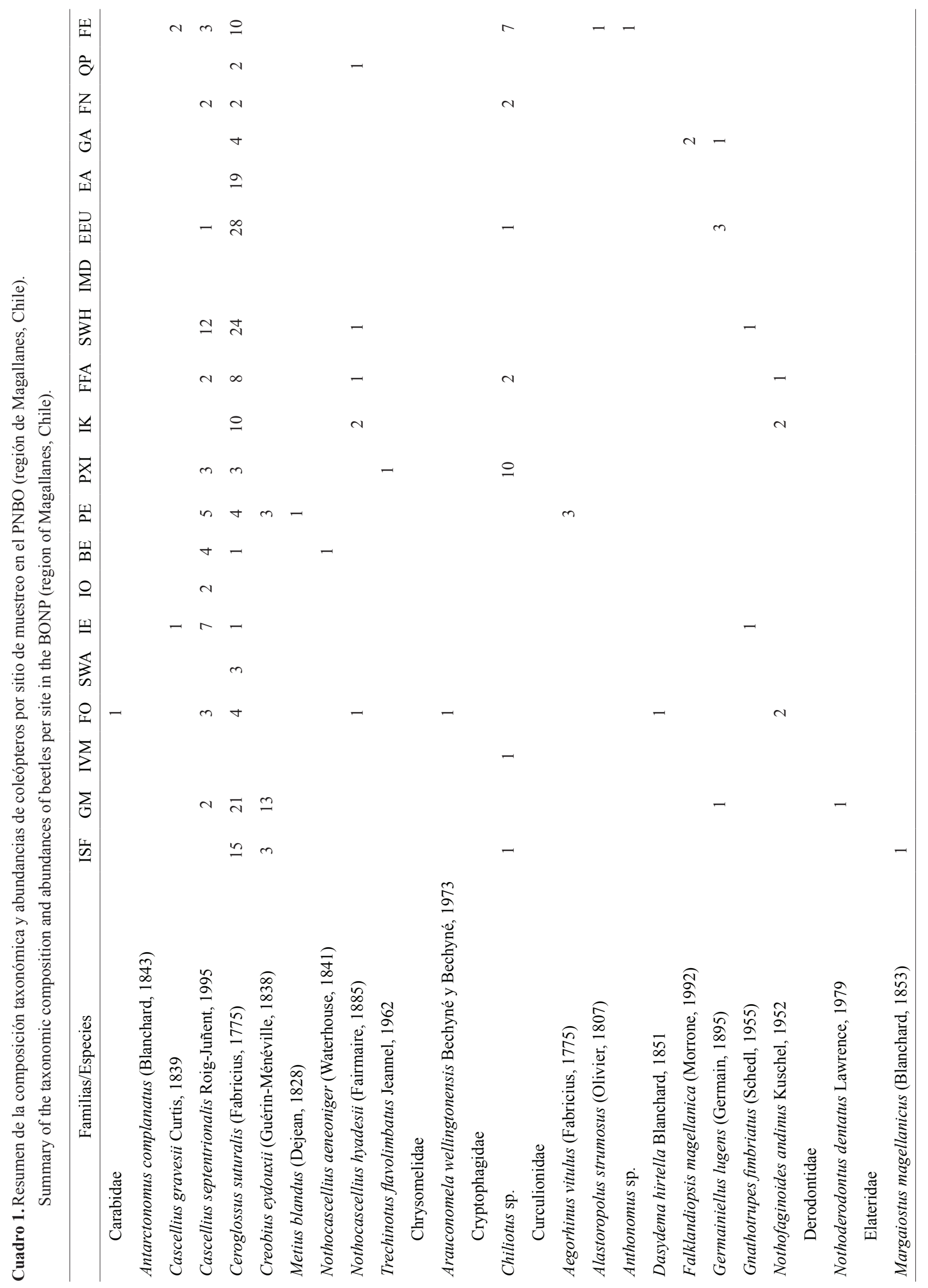


BOSQUE 38(2): 285-297, 2017

Coleópteros del Parque Nacional Bernardo O'Higgins

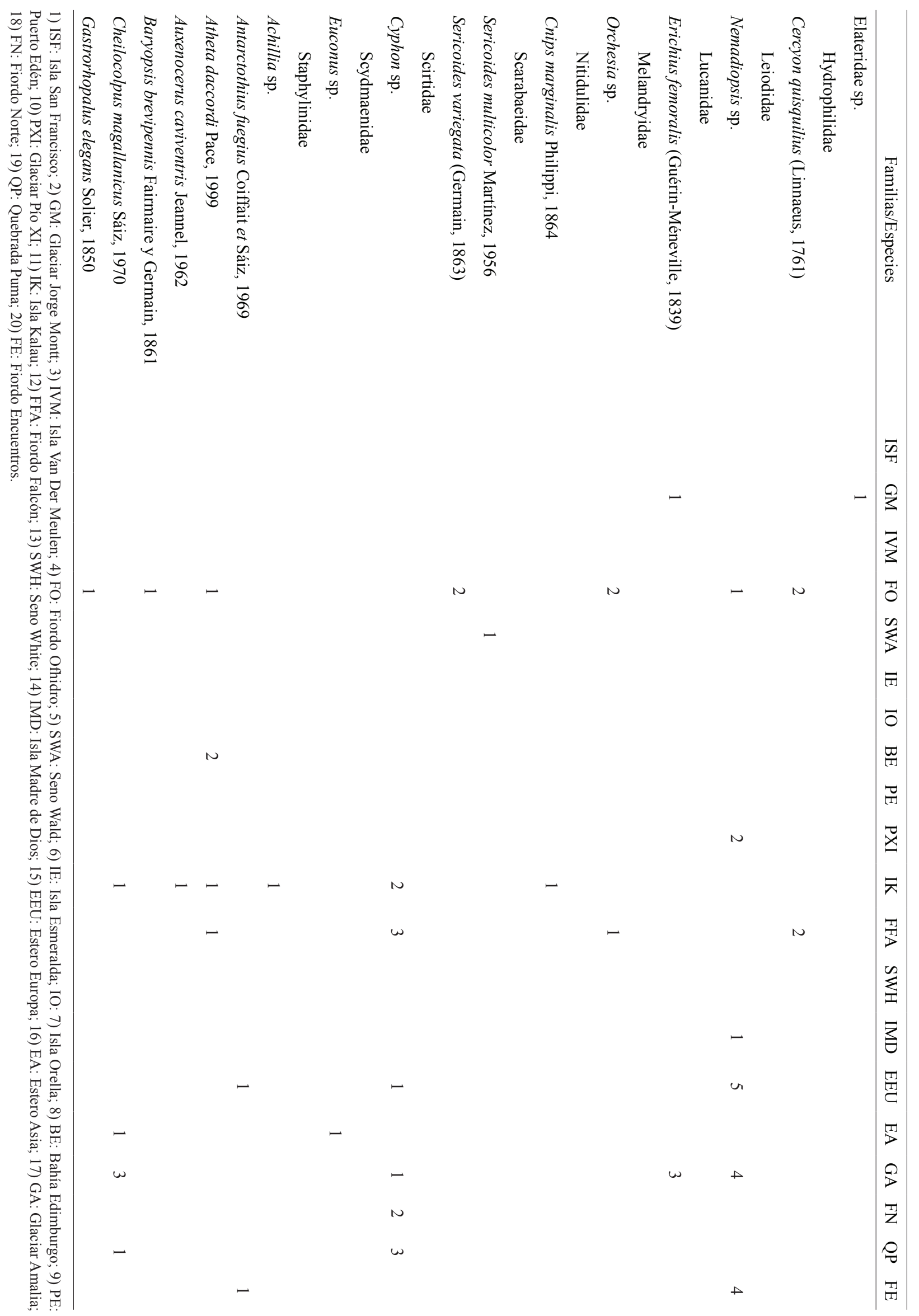


Cuadro 2. Resumen de la composición taxonómica y abundancias de coleópteros por formación vegetacional en el PNBO (región de Magallanes, Chile).

Summary of the taxonomic composition and abundances of beetles per vegetation formation in the BONP (region of Magallanes, Chile).

\begin{tabular}{|c|c|c|c|c|c|}
\hline \multirow{2}{*}{ Familia } & \multirow{2}{*}{ Especie } & \multicolumn{3}{|c|}{ Formaciones vegetacionales } & \multirow{2}{*}{ Total } \\
\hline & & Bosque & Matorral & Turbera & \\
\hline \multirow[t]{9}{*}{ Carabidae $(\mathrm{n}=237)$} & Antarctonomus complanatus & 1 & 0 & 0 & 1 \\
\hline & Cascellius gravesii & 3 & 0 & 0 & 3 \\
\hline & Cascellius septentrionalis & 29 & 12 & 5 & 46 \\
\hline & Ceroglossus suturalis & 83 & 62 & 14 & 159 \\
\hline & Creobius eydouxi & 3 & 16 & 0 & 19 \\
\hline & Metius blandus & 0 & 1 & 0 & 1 \\
\hline & Nothocascellius aeneoniger & 0 & 1 & 0 & 1 \\
\hline & Nothocascellius hyadessi & 4 & 2 & 0 & 6 \\
\hline & Trechinotus flavolimbatus & 1 & 0 & 0 & 1 \\
\hline Chrysomelidae $(\mathrm{n}=1)$ & Arauconomela wellingtonensis & 1 & 0 & 0 & 1 \\
\hline Cryptophagidae $(\mathrm{n}=24)$ & Chiliotus sp. & 23 & 0 & 1 & 24 \\
\hline \multirow[t]{8}{*}{ Curcullionidae $(\mathrm{n}=20)$} & Aegorhinus vitulus & 0 & 3 & 0 & 3 \\
\hline & Alastoropolus strumosus & 1 & 0 & 0 & 1 \\
\hline & Anthonomus sp. & 1 & 0 & 0 & 1 \\
\hline & Dasydema hirtella & 1 & 0 & 0 & 1 \\
\hline & Falklandius magellanicus & 1 & 1 & 0 & 2 \\
\hline & Germainiellus lugens & 0 & 5 & 0 & 5 \\
\hline & Gnathotrupes fimbriata & 2 & 0 & 0 & 2 \\
\hline & Nothofaginoides andinus & 2 & 2 & 1 & 5 \\
\hline Derodontidae $(\mathrm{n}=1)$ & Nothoderodontus dentatus & 0 & 1 & 0 & 1 \\
\hline \multirow[t]{2}{*}{ Elateridae $(\mathrm{n}=2)$} & Elateridae sp. & 0 & 1 & 0 & 1 \\
\hline & Margaiostus magellanicus & 1 & 0 & 0 & 1 \\
\hline Hydrophilidae $(\mathrm{n}=4)$ & Cercyon quisquilius & 3 & 1 & 0 & 4 \\
\hline Leiodidae $(n=17)$ & Nemadiopsis sp. & 13 & 2 & 2 & 17 \\
\hline Lucanidae $(\mathrm{n}=4)$ & Erichius femoralis & 3 & 1 & 0 & 4 \\
\hline Melandryidae (n=3) & Orchesia sp. & 0 & 2 & 1 & 3 \\
\hline Nitidulidae $(\mathrm{n}=1)$ & Cnips marginalis & 0 & 1 & 0 & 1 \\
\hline \multirow[t]{2}{*}{ Scarabaeidae $(\mathrm{n}=3)$} & Sericoides multicolor & 1 & 0 & 0 & 1 \\
\hline & Sericoides variegatus & 0 & 2 & 0 & 2 \\
\hline Scirtidae $(n=12)$ & Cyphon sp. & 8 & 2 & 2 & 12 \\
\hline Scydmaenidae $(\mathrm{n}=1)$ & Euconus sp. & 0 & 1 & 0 & 1 \\
\hline \multirow[t]{7}{*}{ Staphylinidae $(\mathrm{n}=17)$} & Achilia sp. & 0 & 1 & 0 & 1 \\
\hline & Antarctothius fuegius & 1 & 0 & 1 & 2 \\
\hline & Atheta daccordi & 4 & 1 & 0 & 5 \\
\hline & Auxenocerus caviventris & 1 & 0 & 0 & 1 \\
\hline & Baryopsis brevipennis & 1 & 0 & 0 & 1 \\
\hline & Cheilocolpus magallanicus & 2 & 4 & 0 & 6 \\
\hline & Gastrorhopalus elegans & 0 & 1 & 0 & 1 \\
\hline 15 familias & 38 especies & 194 & 126 & 27 & \\
\hline
\end{tabular}


Cuadro 3. Resultados de PERMANOVA sobre el efecto del tipo de ambiente (insular, continental) y formación vegetal (bosque, matorral, turbera) sobre los parámetros comunitarios Riqueza (S), Abundancia (N), Exponencial de Shannon (exp H'), Inverso de GiniSimpson (1/1- $\lambda$ ') y la composición taxonómica considerando abundancias (Bray-Curtis) y presencia/ausencia (Sørensen) de especies.

Results from PERMANOVA for differences in number of species (S), abundance (N), Exponential of Shannon (exp H'), inverse of GiniSimpson (1/1- $\left.\lambda^{\prime}\right)$, and taxonomic composition based on abundances (Bray-Curtis) and presence/absence (Sørensen), on type of environment (insular, continental) and vegetation.

\begin{tabular}{|c|c|c|c|c|c|c|c|c|c|}
\hline \multirow{3}{*}{ Fuente } & \multirow{3}{*}{ g.1. } & \multicolumn{8}{|c|}{ Parámetros comunitarios } \\
\hline & & \multicolumn{4}{|c|}{ Riqueza (S) } & \multicolumn{4}{|c|}{ Abundancia (N) } \\
\hline & & SS & MS & Pseudo-F & $\mathrm{P}(\mathrm{MC})$ & SS & MS & Pseudo-F & $\mathrm{P}(\mathrm{MC})$ \\
\hline Ambiente & 1 & 5,024 & 5,024 & 1,110 & 0,303 & 1,028 & 1,028 & 0,0127 & 0,911 \\
\hline Vegetación & 2 & 8,803 & 4,402 & 0,973 & 0,393 & 59,33 & 29,665 & 0,366 & 0,696 \\
\hline Amb x Veg & 2 & 8,528 & 4,264 & 0,942 & 0,404 & 166,88 & 83,441 & 1,029 & 0,371 \\
\hline Residuos & 26 & 117,65 & 4,525 & & & $2.106,7$ & 81,028 & & \\
\hline Total & 31 & 153,47 & & & & $2.570,2$ & & & \\
\hline
\end{tabular}

\begin{tabular}{|c|c|c|c|c|c|c|c|c|c|}
\hline & & & encial c & hannon (ex & & & de Gi & impson (1) & \\
\hline & & SS & MS & Pseudo-F & $\mathrm{P}(\mathrm{MC})$ & SS & MS & Pseudo-F & $\mathrm{P}(\mathrm{MC})$ \\
\hline Ambiente & 1 & 3,267 & 3,267 & 1,073 & 0,313 & 2,990 & 2,990 & 1,177 & 0,288 \\
\hline Vegetación & 2 & 3,805 & 1,902 & 0,625 & 0,544 & 2,418 & 1,209 & 0,476 & 0,626 \\
\hline Amb $\times$ Veg & 2 & 9,473 & 4,737 & 1,556 & 0,228 & 7,101 & 3,550 & 1,397 & 0,269 \\
\hline Residuos & 26 & 79,141 & 3,044 & & & 66,069 & 2,541 & & \\
\hline Total & 31 & 103,32 & & & & 84,442 & & & \\
\hline
\end{tabular}

\begin{tabular}{|c|c|c|c|c|c|c|c|c|c|}
\hline & & & & & Composic & xonómica & & & \\
\hline & & & Bray & Curtis & & & & sen & \\
\hline & & SS & MS & Pseudo-F & $\mathrm{P}(\mathrm{MC})$ & SS & MS & Pseudo-F & $\mathrm{P}(\mathrm{MC})$ \\
\hline Ambiente & 1 & $1.314,4$ & $1.314,4$ & 0,476 & 0,847 & $1.113,4$ & $1.113,4$ & 0,413 & 0,876 \\
\hline Vegetación & 2 & $4.296,5$ & $2.148,3$ & 0,779 & 0,671 & $4.977,0$ & $2.488,5$ & 0,922 & 0,514 \\
\hline Amb x Veg & 2 & $4.630,7$ & $2.315,3$ & 0,839 & 0,611 & $3.827,0$ & $1.913,5$ & 0,709 & 0,741 \\
\hline Residuos & 26 & 71.727 & $2.758,7$ & & & $70.143,0$ & $2.697,8$ & & \\
\hline Total & 31 & $84.430,0$ & & & & $81.989,0$ & & & \\
\hline
\end{tabular}

Valores de probabilidad basados en un suma de cuadrados tipo III utilizando 50.000 permutaciones de residuos bajo un modelo reducido. P(MC): valor de $P$ obtenido utilizando el algoritmo de Monte Carlo.

ros registrados en el PNBO y sectores aledaños, es que varias de sus especies pertenecen a géneros monotípicos. Las especies de Carabidae, Curculionidae, Crytophagidae y Derodontidae son típicas de bosques hidrófilos en asociación con Nothofagus betuloides (Mib.) Oerst. y N. pumilio (Poepp. et Endl.) Krasser y toda esta biota presenta una estrecha relación a su vez con la historia geomorfológica del paisaje sur-occidental de los Andes (Morrone 2006, Jerez y Muñoz-Escobar 2015).

Al evaluar la diversidad de coleópteros en los sitios prospectados, no se evidenció una diferenciación o estructuración con respecto a ninguno de los parámetros comu- nitarios analizados $\left(\mathrm{S}, \mathrm{N}\right.$, exp $\left.\mathrm{H}^{\prime}, 1 / 1-\lambda^{\prime}\right)$ o composición taxonómica, ya sea considerando los factores del tipo de vegetación o tipo de ambiente. Lo anterior respondería a un factor más bien histórico - biogeográfico, ya que el PNBO se encuentra principalmente asociado a la provincia biogeográfica del Páramo Magallánico (Morrone 2006), el cual representa una biota ancestral única producto de una serie de eventos geomorfológicos acaecidos en la zona (Crisci et al. 1991). Precisamente, los continuos avances y retrocesos de los glaciares, resultó en una fauna terrestre extraordinariamente pobre en número de especies comparada con zonas ecológicamente similares (Jerez y 

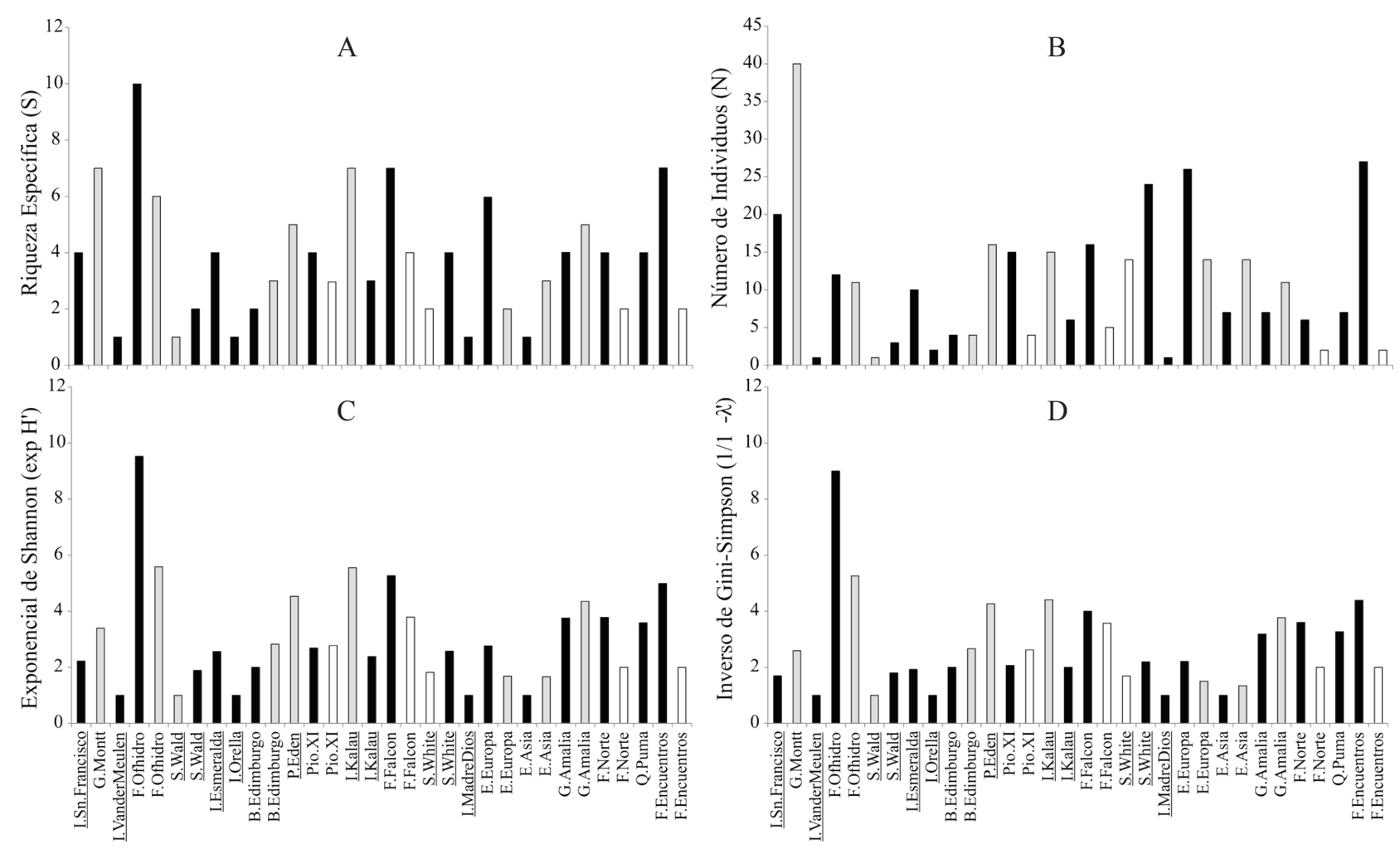

Figura 2. Resultados de parámetros comunitarios por unidad experimental de A) riqueza específica, B) abundancia, C) exponencial de Shannon y D) inverso de Gini-Simpson. Barras negras representan sectores de Bosque, barras grises representan Matorrales y barras blancas Turberas. Nombres de sectores insulares están subrayados.

Results of community index for experimental unit: A) Richness, B) Abundance, C) Exponential of Shannon and D) Inverse of Gini-Simpson. Black, Gray and White bars represent forest, scrub and peatland respectively. Names of insular sectors are underlined.

Muñoz-Escobar 2015), lo que explicaría el bajo número de especies registradas en el PNBO. Además, MuñozEscobar et al. (2015) señalan que sólo al considerar una escala de análisis de provincia biogeográfica (según Morrone 2006), se observa una mayor estructuración en la diversidad de coleópteros en comparación a lo esperado por azar. Lo anterior, indica que escalas de paisaje menores (e.g. formaciones vegetacionales) tienen un aporte menor a lo esperado para la diversidad regional de coleópteros para Sudamérica austral.

El hecho de que el PNBO presente un solo gran ensamblaje, permite analizar su biota como un todo, lo cual facilita la generación de planes de manejo y gestión del parque según la futura Ley del Servicio de Biodiversidad y Áreas Protegidas (MMA 2015). Al respecto, de las 38 especies registradas, once de ellas serían especies que están asociadas significativamente (figura 4) y, por tanto, pueden ser el foco para futuros estudios entomológicos en la zona. Entre estas, se destacan las especies de Carabidae (C. suturalis, C. septentrionalis, C. eydouxii y $N$. hyadesii), todas de hábitos principalmente hidrófilos asociadas a los bosques subantárticos (Roig-Juñent 2000, Jiroux 2006). Debido a su particular rango de distribución (sur-occidental de Los Andes), estas especies serían excelentes candidatas para estudios de filogeografía y evaluar áreas como fuente de diversidad (e.g. refugios) y patrones de recolonización postglacial.

Una comparación con la revisión de Jerez y MuñozEscobar (2015) sobre coleópteros del Páramo Magallánico de la región de Magallanes, muestra que al menos 18 especies de las familias Apionidae (3), Byrrhidae (1), Carabidae (6), Chrysomelidae (2), Curculionidae (2 ) y Lucanidae (1) no fueron registradas en el presente estudio. Por otra parte, en este trabajo se incorporan nuevos registros de especies de coleópteros, principalmente de sectores asociados a la provincia del Bosque Magallánico (Cryptophagidae: 1, Curculionidae: 4, Derodontidae: 1, Elateridae: 2, Lucanidae: 1, Melandryidae: 1, Nitidulidae: 1, Scarabeidae: 1, Scirtidae: 1, Scydmaenidae: 1 y Staphylinidae: 5). Uno de los motivos que podría explicar estas diferencias, sería el método de muestreo utilizado en este estudio, ya que la eficiencia de las trampas depende del efecto que tienen algunas variables atmosféricas sobre la actividad de los individuos (Koivula et al. 2003). Para el caso de este trabajo, las trampas estuvieron en cada sitio de muestreo un período muy breve de tiempo ( 24 horas), 


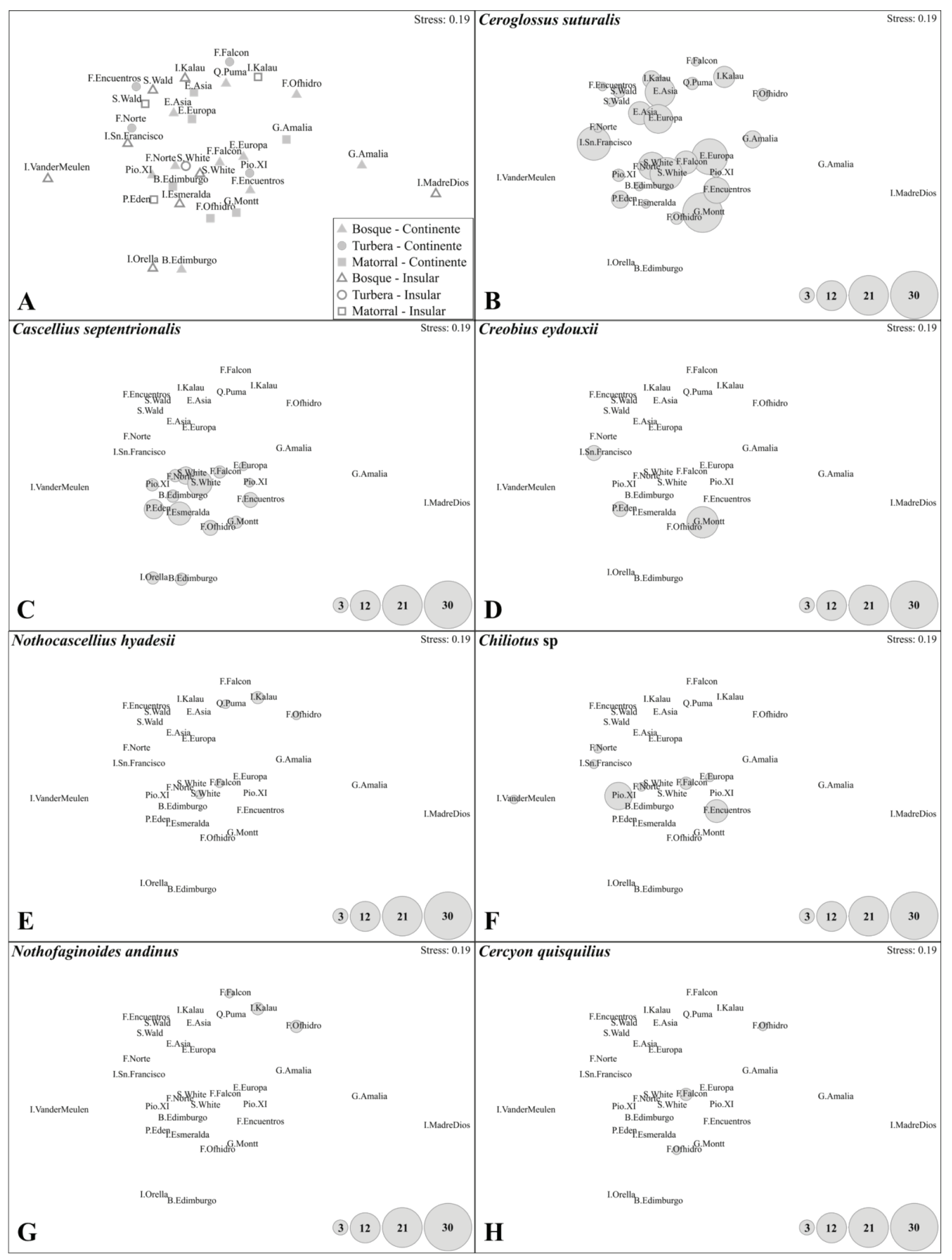

Figura 3 (continúa). 


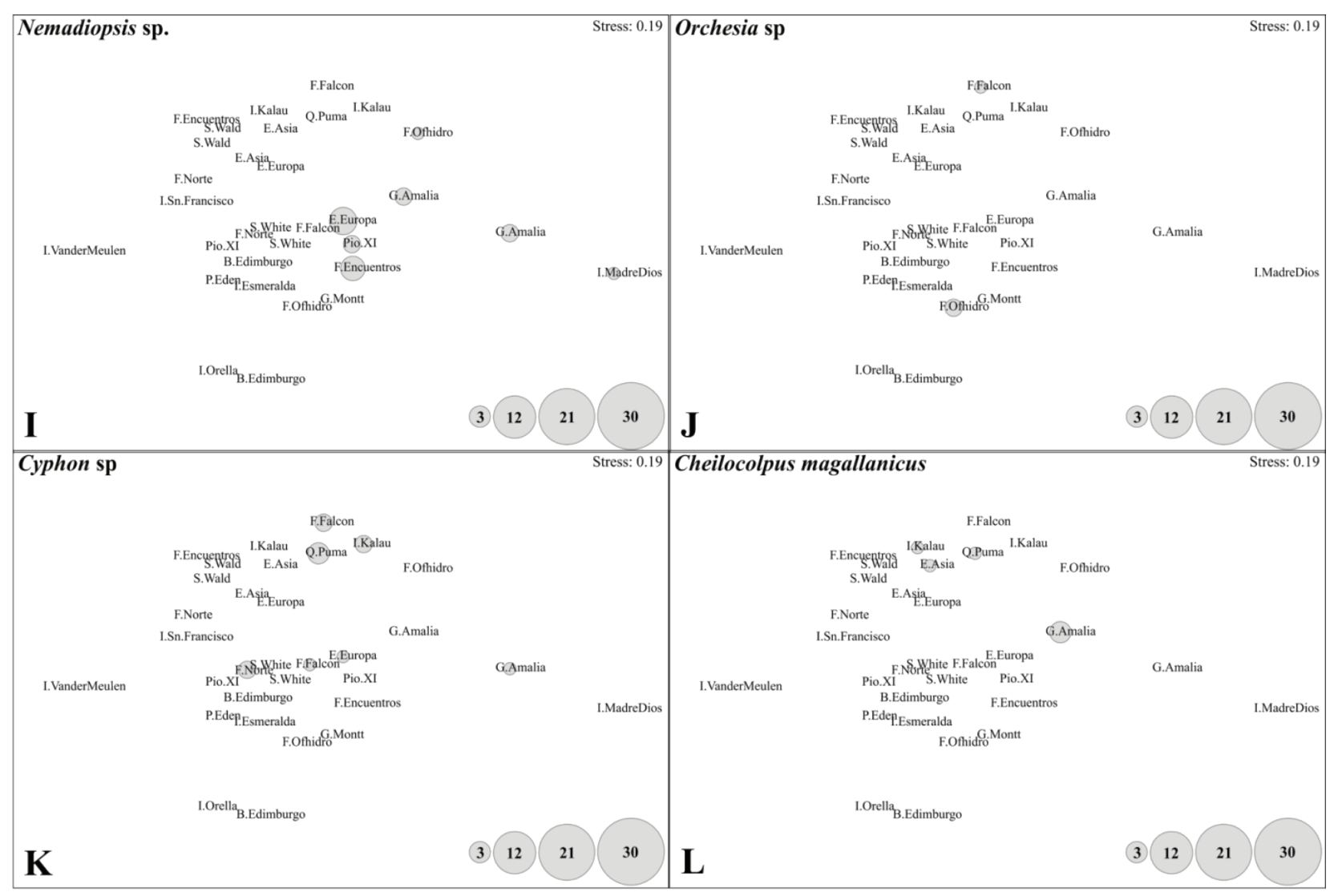

Figura 3. Escalamiento multidimensional no paramétrico (nMDS). A) Ordenamiento de las distintas unidades experimentales según el total de la composición de especies utilizando el índice de similitud de Bray-Curtis. B-L) Diámetro de los círculos indica las abundancias para las especies que explican el patrón global de coleópteros del PNBO.

Nonparametric Multidimensional Scaling (nMDS). A) Ordination among experimental units according to the total species composition using the Bray-Curtis similarity index. B-L) Diameter circle indicates the abundance of key species that explain the overall pattern of beetles in the BONP.

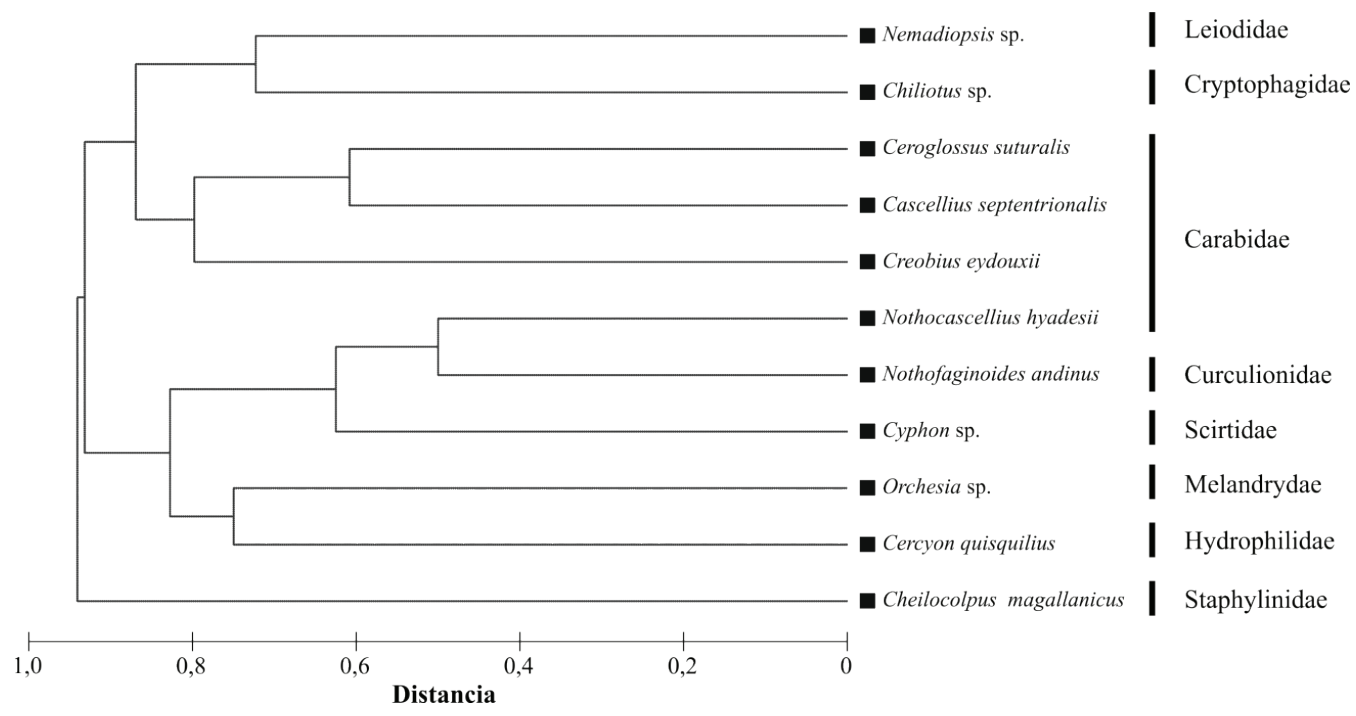

Figura 4. Dendrograma (modo-R) obtenido a partir del algoritmo de encadenamiento o vinculación promedio de las 11 especies de coleópteros determinados por BVSTEP a partir del índice de asociación de Whittaker. Símbolo cuadrado muestra la presencia de un solo "grupo coherente" $(P<0,05)$ según la prueba SIMPROF tipo 3.

Dendrogram from (r-mode) group-average clustering of the 11 'most important' species by BVSTEP algorithm, based on Whittaker's Index of Association among species. Square symbol denotes one coherent group which was significantly differentiated by Type 3 SIMPROF tests $(P<0.05)$. 
debido a temas logísticos y factibilidad de permanencia en cada sitio prospectado. A pesar de esto y en general, la composición taxonómica de coleópteros registrada corresponde a lo descrito en la literatura para la región de Magallanes y para el Páramo Magallánico (Jeannel 1962, Morrone et al. 1994, Roig-Juñent 2000, Jiroux 2006). Si bien se enfrentaron problemas de permanencia en cada sitio muestreado, destaca que allí también radica el aporte de este trabajo, ya que se entrega información de especies para lugares prácticamente inexplorados científicamente, principalmente de sectores insulares (e.g. Isla Kalau, Isla San Francisco).

Del punto de vista de la conservación etno-científica (Martinic 2011) y dada la activa participación que tendrán las comunidades de pueblos originarios en áreas protegidas, se considera importante que la comunidad kawésqar se involucre en la difusión del conocimiento de la entomofauna del sector, especialmente en sectores donde las comunidades rurales e indígenas que allí habitan deben compartir los beneficios y responsabilidades de las áreas protegidas (Pauchard y Villarroel 2002). En este sentido, durante el transcurso de este estudio se logró encontrar que los kawéskar, denominan a las especies $C$. suturalis $(\mathrm{Ca}-$ rabidae) y E. femoralis (Lucanidae) "æk asar" y "fqalto" respectivamente. Mientras que coleópteros Curculionidae en general (burritos) son llamados "kekiasqe" y los Elateridae (saltapericos o hacheros) "camančok". Cabe señalar que la gran mayoría de las especies registradas, dado su tamaño y por tanto dificultad de observar, no disponen de nombre etno-vernacular.

Finalmente, la información entregada en este trabajo permite evaluar patrones y procesos metacomunitarios de coleópteros que sirven de base para otras investigaciones de carácter biogeográfico. De hecho, estudios de biogeografía histórica constituyen uno de los componentes básicos de cualquier tipo de medida o manejo para el establecimiento y selección de áreas a ser protegidas (Morrone et al. 1994). Lo anterior cobra importancia al considerar que la legislación chilena reconoce la protección de ecosistemas y procesos evolutivos como uno de los principales objetivos del Sistema Nacional de Áreas Protegidas del Estado (SNASPE) (Pauchard y Villarroel 2002) y del Proyecto Servicio de Biodiversidad y Áreas Protegidas y el Sistema Nacional de Áreas Protegidas (MMA 2015).

\section{CONCLUSIONES}

De acuerdo con las especies capturadas (38) en el PNBO, las familias Carabidae, Curculionidae y Staphylinidae presentan la mayor riqueza específica. Entre los coleópteros destacan los carábidos $C$. suturalis y $C$. septentrionalis, los cuales además de estar presentes en todas las formaciones vegetacionales, también son los más abundantes dentro del parque. No se observa estructuración de coleópteros en función de las formaciones vegetacionales ni tipos de ambientes, tanto en términos de descriptores comunitarios como composición taxonómica. Los resultados obtenidos señalan que 11 especies describen el patrón de ordenamiento presente en el parque; estas pertenecen a las familias Carabidae (4), Cryptophagidae (1), Curculionidae (1), Hydrophilidae (1), Leiodidae (1), Melandryidae (1), Scirtidae (1) y Staphylinidae (1). Cabe destacar que estas 11 especies representan una sola asociación, es decir, covarian coherentemente en todo el PNBO. Este resultado facilitará el foco de próximos planes de manejo que se puedan realizar dentro del parque, ya sea en el marco del Servicio de Biodiversidad y Áreas Protegidas y/o monitoreos medioambientales para evaluar el estado de conservación de los tipos de ambientes en el PNBO.

\section{AGRADECIMIENTOS}

Al Proyecto INNOVA CORFO 08CTU01-20 “Caracterización territorial del Parque Nacional Bernardo O’Higgins, su potencial económico, turístico, científico y cultural". Este proyecto fue mandatado por CONAF Magallanes y ejecutado por el Centro de Estudios del Cuaternario (CEQUA). Se agradece también al Proyecto DIUC $\mathrm{N}^{\circ}$ 212.113.080-1.0 de la Universidad de Concepción por financiar parte de este trabajo. Finalmente a Francisco Arroyo Sotomayor por entregar los nombres Kawésqar de los coleópteros y a Carolina Galleguillos, Kary Haro y Soledad Contreras por su apoyo en el trabajo de terreno.

\section{REFERENCIAS}

Anderson MJ, RN Gorley, KR Clarke. 2008. PERMANOVA+ for PRIMER: Guide to software and statistical methods. Plymouth, United Kingdom. PRIMER-E. 214 p.

CEQUA (Centro de Estudios del Cuaternario, Fuego-Patagonia y Antártica, CL). 2011. Parque Nacional Bernardo O'Higgins. Punta Arenas, Chile. La Prensa Austral Impresos. 248 p.

Clarke KR, RN Gorley. 2006. PRIMER v6: User manual/Tutorial. Plymouth, United Kingdom. PRIMER-E. 190 p.

Clarke KR, PJ Somerfield, MG Chapman. 2006. On resemblance measures for ecological studies, including taxonomic dissimilarities and a zero-adjusted Bray-Curtis coefficient for denuded assemblages. Journal of Experimental Marine Biology and Ecology 330: 55-80.

Clarke KR, RM Warwick. 1998. Quantifying structural redundancy in ecological communities. Oecologia 113: 278-289.

Crisci JV, MM Cigliano, JJ Morrone, S Roig-Juñent. 1991. Historical biogeography of southern South America. Systematic Zoology 40(2): 152-171.

Elgueta M. 2008. Holometábolos, Orden Coleoptera. In Comisión Nacional del Medio Ambiente eds. Biodiversidad de Chile: Patrimonio y Desafíos. Segunda Edición Actualizada. Santiago, Chile. Ocho Libros Editores. p. 144-150.

Jeannel R. 1962. Les Silphidae, Leiodidae, Camiaridae et Catopidae de Paleantarctide occidentale. In Delamare CL, E Rapoport eds. Biologie de l`Amérique australe. Volumen I. Paris, France. Éditions du Centre National de la Recherche Scientifique. p. 481-525.

Jerez V, C Muñoz-Escobar. 2015. Coleópteros y otros insectos 
asociados a turberas del páramo magallánico en la Región de Magallanes, Chile. Cap. 7. In Domínguez E, D Vega-Valdés eds. Funciones y servicios ecosistémicos de las turberas en Magallanes. Punta Arenas, Chile. Colección Libros INIA 33. p. 199-240.

Jerez V, A Zúñiga-Reinoso, C Muñoz-Escobar y J Pizarro-Araya. 2015. Acciones y Avances sobre la conservación de insectos en Chile. Gayana 79(1): 1-3.

Jiroux E. 2006. Le genre Ceroglossus. Vol. 14. Magellanes. Paris, France. Collection Systématique. 173 p.

Jorquera-Jaramillo C, JM Alonso Vega, J Aburto, K Martínez-Tillería, MF León, MA Pérez, CF Gaymer, FA Squeo. 2012. Conservación de la biodiversidad en Chile: Nuevos desafíos y oportunidades en ecosistemas terrestres y marinos costeros. Revista Chilena de Historia Natural 85: 267-280.

Jost L. 2006. Entropy and diversity. Oikos 113: 363-375.

Koivula M, DJ Kotze, L Hiisivuori, H Rita. 2003. Pitfall trap efficiency: do trap size, collecting fluid and vegetation structure matter? Entomologica Fennica 14: 1-14.

Martinic BM. 2011. Ethnogeographic guide to the Bernardo O'Higgins National Park. Magallania 39(2): 318-320.

MMA (Ministerio del Medio Ambiente, CL). 2011. Ley N 19.300 sobre Bases Generales del Medio Ambiente. Ley Orgánica de Superintendencia del Medio Ambiente. Consultado 18 ene. 2017. Disponible en http://www.munistgo.info/medio ambiente/biblioteca_digital/Ley19300_12_2011.pdf

MMA (Ministerio del Medio Ambiente, CL). 2015. Proyecto de Ley que crea el Servicio de Biodiversidad y Áreas Protegidas. Consultado 18 ene. 2017. Disponible en http://portal. $\mathrm{mma}$. gob.cl/proyecto-de-ley-que-crea-el-servicio-de-biodiversidad-y-areas-protegidas/

MINSEGPRES (Ministerio Secretaria General de la Presidencia, CL). 2010. Ley 20.417. Crea el Ministerio, el Servicio de Evaluación ambiental y la Superintendencia del medio ambiente. Consultado 18 ene. 2107. Disponible en http://www.
leychile.cl/Navegar?idNorma=1010459

Morrone JJ. 2006. Biogeographic areas and transition zones of Latin America and the Caribbean Islands, based on panbiogeographic and cladistic analyses of the entomofauna. Annual Review of Entomology 51: 467-494.

Morrone JJ, S Roig-Juñent, JV Crisci. 1994. South American Beetles. National Geographic Research \& Exploration 10 (1): 104-115.

Muñoz-Escobar C, S Roig-Juñent, R Carrara, V Jerez. 2015. Scale analysis of carabids richness (Coleoptera: Carabidae) in southern South America. Gayana 79(2): 167-172.

Pauchard A, P Villarroel. 2002. Protected Areas in Chile: History, Current Status, and Challenges. Natural Areas Journal 22(4): 318-330.

Pisano E. 1977. Fitogeografía de Fuego-Patagonia Chilena. I Comunidades vegetales entre las latitudes $52^{\circ}$ y $56^{\circ} \mathrm{S}$. Anales del Instituto de la Patagonia 8: 121-250.

Roig-Juñent S. 2000. The subtribes and genera of the tribe Broscini (Coleoptera: Carabidae). Cladistic analysis, taxonomic treatment and biogeographical considerations. Bulletin of the American Museum of Natural History 255: 1-90.

Shannon C. 1948. A mathematical theory of communication. The Bell System Technical Journal 27: 379-423.

Simpson EH. 1949. Measurement of diversity. Nature 163: 688.

Somerfield PJ, KR Clarke. 2013. Inverse analysis in non-parametric multivariate analyses: distinguishing groups of associated species which covary coherently across samples. Journal of Experimental Marine Biology and Ecology 449: 261-273.

Sørensen T. 1948. A method of establishing groups of equal amplitude in plant sociology based on similarity of species and its application to analyses of the vegetation on Danish commons. Biologiske Skrifter 5: 1-34.

Whittaker RH. 1952. A study of summer foliage insect communities in the Great Smoky Mountains. Ecological Monographs 22: 1-44. 
